\title{
Análisis del paisaje para la evaluación ecológica rápida de alternativas de relocalización de una ciudad devastada
}

\author{
Landscape analysis for rapid ecological assessment of relocation alternatives for a \\ devastated city
}

\author{
FRANCISCO DE LA BARRERA ${ }^{1,2,3}$, SONIA REYES-PAECKE ${ }^{4, ~ * ~ \& ~ L U I S ~ M E Z A ~} 4$ \\ ${ }^{1}$ Cienciambiental Consultores S.A., Almirante Zegers 814-B, Santiago, Chile \\ ${ }^{2}$ Facultad de Geografía e Historia, Universidad de Barcelona, Montalegre 6, Barcelona, España \\ ${ }^{3}$ Centro Nacional del Medio Ambiente, Universidad de Chile, Larraín 9975, Santiago, Chile \\ ${ }^{4}$ Instituto de Estudios Urbanos y Territoriales, Pontificia Universidad Católica de Chile, El Comendador 1916, Santiago, \\ Chile \\ * Autor correspondiente: sreyespa@uc.cl
}

\begin{abstract}
RESUMEN
Tras la erupción del volcán Chaitén (Chile) y la destrucción de la ciudad homónima, tres localidades resultaron ser alternativas oficiales para la relocalización de la ciudad. Fandango, Santa Bárbara y Bahía Pumalín fueron evaluadas en un corto plazo a partir de diversos criterios, siendo uno de ellos el potencial impacto de la nueva urbanización sobre la biodiversidad local, a través del análisis del paisaje como herramienta. Los atributos del paisaje evaluados, sobre una imagen satelital LANDSAT 5 (noviembre 17, 2008), fueron (1) composición y representación de cada tipo de ecosistema, (2) conectividad y (3) valor ambiental a través de la clasificación de los ecosistemas dominantes y su estado sucesional, a partir de su heterogeneidad y grado de madurez del ecosistema. Las métricas obtenidas a través de ArcView ${ }^{\circledR}$ (ESRI) 3.2 y FRAGSTATS, fueron la superficie, el número de parches y el índice de cohesión (IC) de cada tipo de ecosistema. El valor ambiental fue determinado en forma cualitativa e in situ. Entre las tres alternativas, Santa Bárbara fue escogida por las autoridades para la construcción de Nueva Chaitén. Esta localidad presenta más de un $70 \%$ de su superficie cubierta por bosque, un alto grado de conectividad de bosques $(\mathrm{IC}>99.0)$ y matorrales $(\mathrm{IC}>94.0)$, un estado sucesional avanzado, una alta heterogeneidad (en especies y estratos) y un alto valor ambiental hacia el interior. Hacia la costa, Santa Bárbara presenta sectores antropizados, conserva un estado sucesional avanzado y posee un valor medio-alto de heterogeneidad y valor ambiental. Ante la inminente urbanización de esta localidad, se recomendó la mantención de la integridad de los sectores aledaños al estero Santa Bárbara y la continuidad de los bosques, en especial hacia el oriente de la Ruta 7. El método demostró, tras su aplicación: (1) ser útil para evaluar la biodiversidad en condiciones de falta de información y recursos para generar información a nivel de especies, (2) ser fácilmente replicable, dado que las categorías utilizadas (tipos de ecosistemas) son estandarizables y las instituciones públicas tienen facilidades para acceder a imágenes satelitales $\mathrm{y}$, finalmente, (3) permitir la incorporación de criterios ecológicos en fases tempranas de la toma de decisiones en el ámbito del ordenamiento territorial y de la planificación urbana.
\end{abstract}

Palabras clave: cambio uso de suelo, Chaitén, ecología urbana, evaluación ambiental, urbanización.

\begin{abstract}
After the eruption of the Chaitén Volcano (Chile) and the destruction of the neighboring Chaitén city, some alternative sites were evaluated for the relocation of administrative facilities and housing for the displaced. Three towns (Fandango, Santa Bárbara and Bahía Pumalín) were evaluated by means of a multi-criteria analysis, including the impact that a new urban development would have on local biodiversity. This impact was evaluated through a landscape analysis. The landscape attributes studied were (1) composition and representation of each ecosystem type, (2) connectivity, and (3) environmental value, based on a classification of the prevailing ecosystems and their respective stages of succession, defined as a function of ecosystem heterogeneity and maturity. Landscape metrics were calculated from LANDSAT 5 satellite imagery (November 17th, 2008) with ArcView ${ }^{\circledR} 3.2$ (ESRI) and Fragstats, including the surface cover of each ecosystem type, the number of patches and the cohesion index (CI). Environmental value was determined qualitatively in situ. Santa Barbara was selected by authorities as the best place for New Chaitén City. Over $70 \%$ of this is area is covered by forest, achieving a high degree of forest connectivity (CI >99.0) and shrubs (CI > 94.0), with an advanced stage of succession, a high level of heterogeneity (species and strata) and a high level of inland environmental value. On the coast, Santa Bárbara has anthropic influence, but maintains an advanced state of succession, a high level of heterogeneity, and a medium-high degree of environmental value. Regarding the future urban development at this location, the recommendations were: maintenance of riverside integrity and the continuity of forests in Santa
\end{abstract}


Bárbara. The proposed methodology proved to be: (1) useful for biodiversity assessment, despite the lack of information at species level, (2) easily replicable, due to the use of standard categories (ecosystem types) and easier access to satellite images, and (3) able to integrate environmental criteria at early stages of decision making in land use and urban planning processes

Key words: Chaitén, environmental assessment, land use change, urban development, urban ecology.

\section{INTRODUCCIÓN}

El día 2 de mayo de 2008 el volcán Chaitén $\left(42,83^{\circ} \mathrm{S}\right.$ y $\left.72,65^{\circ} \mathrm{O}, 1122 \mathrm{msnm}\right)$, ubicado a 10 $\mathrm{km}$ de la ciudad homónima (Provincia de Chaitén), inició una erupción pliniana, evacuando durante la fase explosiva hasta cuatro $\mathrm{km}^{3}$ de magma riolítico, con una vigorosa pluma de 10.7 a $16.8 \mathrm{~km}$ de altura, y una gran emisión de cenizas (Naranjo \& Stern 2004, Folch et al. 2008, Carn et al. 2009, Castro \& Dingwell 2009, Lara 2009). La totalidad de la población urbana (4625 habitantes) y de aquella asentada en el área definida por dos radios de seguridad, de 30 y $50 \mathrm{~km}$, fue evacuada, siendo localizada en forma temporal en Puerto Montt y Castro (en la Isla de Chiloé), principalmente (Lara 2009). Diez días más tarde, el desprendimiento de parte del domo del volcán ocasionó lahares e inundaciones que dejaron inhabitable la ciudad (Carn et al. 2009, Lara 2009).

En este escenario de desastre, incertidumbre acerca de la evolución futura de la actividad del volcán Chaitén y presión de los ciudadanos por regresar a sus hogares, se evaluó la reconstrucción y/o relocalización de la ciudad. Luego de descartar técnicamente la primera opción, fueron seleccionadas tres alternativas de relocalización (Pontificia Universidad Católica de Chile 2009), las cuales debieron ser evaluadas comparativamente en un corto plazo, para lo cual se utilizó una Evaluación Ambiental Estratégica (EAE) con un análisis multicriterio (AMC).

La EAE sirve para integrar la consideración de potenciales impactos ambientales en el diseño de políticas, planes y programas. Se aplica en fases tempranas del proceso de toma de decisiones, permitiendo la introducción de modificaciones en el diseño de dichas acciones, siendo apropiada para la evaluación de las alternativas de relocalización de una ciudad. El procedimiento de evaluación de la EAE implica la incorporación de objetivos o criterios frente a los cuales se contrastan los potenciales impactos de las políticas, planes o programas (Oñate et al. 2002, Hervé 2010). En Chile, tras la promulgación de Ley 20417 del 26/01/2010 (Ministerio Secretaría General de la Presidencia) se establece la obligatoriedad de que los instrumentos de planificación territorial se sometan a una EAE, no obstante, no se establecen directrices metodológicas.

El AMC es una metodología diseñada para contribuir a la toma de decisiones y consiste básicamente en una serie de técnicas que permiten evaluar alternativas mediante la aplicación de un conjunto de criterios, los cuales son definidos por los múltiples agentes involucrados en el proceso de toma de la decisión (e.g., autoridades políticas, profesionales de servicios públicos, técnicos y científicos; Lahdelma et al. 2000). Las técnicas aplicadas en el presente estudio, corresponden a aquellas denominadas "discretas" porque se aplica a un número limitado de alternativas (Brown et al. 2001). Dado que una evaluación ambiental requiere integrar criterios, el AMC ha sido ampliamente aplicado en EAEs y en planificación territorial (ver Malczewzki et al. 1997, Lahdelma et al. 2000, Brown et al. 2001, Henig \& Weintraub 2006, Liu et al. 2010). Para esta evaluación se definieron criterios sociales, ecológicos, ambientales y económicos (Pontificia Universidad Católica de Chile, 2009).

Para la evaluación de cada uno de los criterios se aplicaron y/o desarrollaron métodos que permitieran hacer uso de la escasa información existente y/o generar datos rápida y oportunamente, para permitir la comparación entre las alternativas de localización. Los criterios ecológicos seleccionados se enfocaron en la evaluación de los impactos que la futura urbanización podría ocasionar sobre ecosistemas terrestres o acuáticos y/o áreas de alto valor ambiental o de biodiversidad, con énfasis en la fragmentación del paisaje y la disminución de hábitats disponibles para las distintas especies (Forman 1995, Grez et al. 2006, Pauchard et al. 2006).

El valor ambiental, desde el punto de vista ecológico, puede representarse a través de la 
biodiversidad contenida en un territorio, siendo un valor a conservar. La biodiversidad se expresa, operacionalmente, en tres niveles: genes, especies y ecosistemas o paisajes, siendo los atributos fundamentales de este último, su composición, estructura y función (Noss 1990, Serey et al. 2007). Complementariamente al análisis de la biodiversidad a través del paisaje, el estudio de la vegetación puede resultar útil como herramienta de gestión ambiental, dado que informa sobre las características globales del paisaje (o tipo de ecosistema), de su madurez en términos de su sucesión ecológica, del grado de intervención antrópica y de la capacidad de albergar fauna nativa (Ausden 2007, Hagar 2007, Adams et al. 2009, Drever \& Martin 2010).

En este contexto, el objetivo del presente trabajo es presentar la metodología elaborada por los autores para evaluar comparativamente localizaciones alternativas de una urbanización, mediante la definición de criterios ecológicos:
(1) continuidad de los ecosistemas terrestres y acuáticos presentes, (2) diversidad estructural y (3) formaciones vegetacionales dominantes. La metodología es especialmente útil para los procesos de toma de decisiones en el ámbito de la planificación, la evaluación ambiental y el ordenamiento territorial. Sus ventajas son: la utilización de información existente y de fácil acceso, bajo consumo de tiempo y recursos monetarios y aplicabilidad en procesos regulares de toma de decisiones que involucran agentes públicos y privados.

\section{MÉTODOS}

Las tres localidades estudiadas se ubican en la costa de la Provincia de Palena $\left(42.70^{\circ} \mathrm{S}\right.$ y $72.40^{\circ}$ O; Fig. 1$)$. La vegetación del territorio está constituida mayoritariamente por el Bosque Siempreverde Templado de Interior, dominado por Nothofagus nitida (Phil.) Krasser (coigüe de Chiloé) y Podocarpus nubigenus Lindl. (mañío macho), con predominio del estrato arbóreo, asociado a zonas frías y a suelos de ñadis -húmedos y de mal drenaje- poco aptos para la

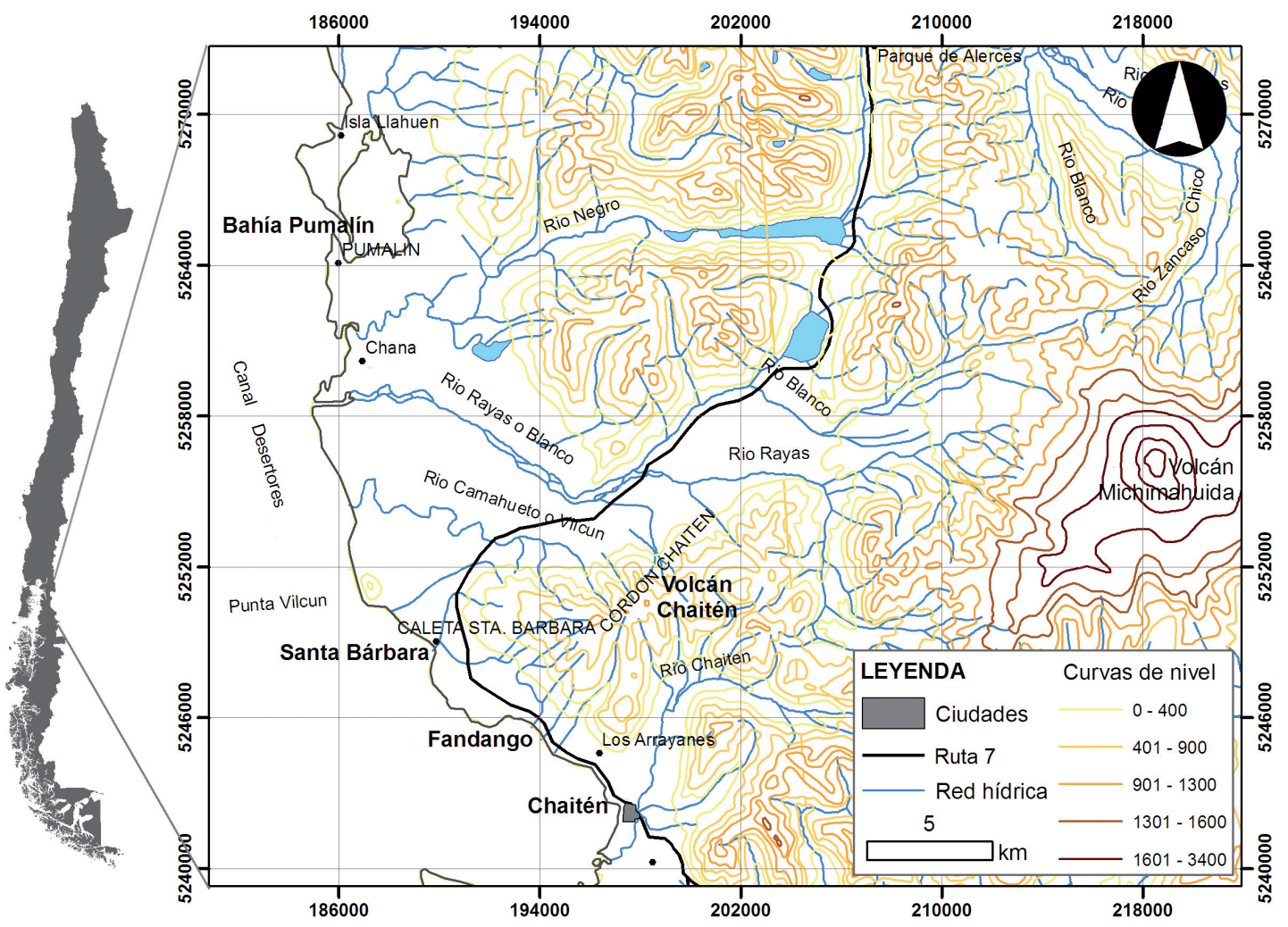

Fig. 1: Ámbito geográfico de las localidades evaluadas como alternativas (Santa Bárbara, Fandango y Bahía Pumalín).

Geographical position of alternatives evaluated locations (Santa Barbara, Fandango and Bahía Pumalín). 
agricultura (Ramírez et al. 1996, Luebert \& Pliscoff 2006). La Región presenta un bajo grado de ocupación humana, razón por la cual predominan los paisajes silvestres.

Para el análisis de paisaje se identificaron los usos de suelo mediante la clasificación de una imagen LANDSAT 5 (resolución $30 \mathrm{~m}$ ) del 17/11/2008, con ArcView ${ }^{\circledR} 3.2$ (ESRI). Los usos de suelo fueron clasificados en (1) áreas urbanas (incluye aeródromos y embarcaderos), (2) terrenos agrícolas, (3) praderas (silvestres y artificiales), (4) matorrales, (matorrales y matorrales arborescentes), (5) bosques (bosque adulto, renovales y bosque/renoval), (6) áreas sin cobertura vegetal y (7) cuerpos de agua (ríos, lagos, lagunas y océano). La clasificación estuvo basada en el "Catastro de Recursos Vegetacionales Nativos de Chile (Corporación Nacional Forestal \& Comisión Nacional de Medio Ambiente 1999)

Los atributos evaluados para analizar el paisaje en cada una de las alternativas fueron: (1) la composición y dominancia de ecosistemas que constituyen el paisaje, (2) la conectividad estructural entre los ecosistemas y (3) el valor ambiental de sus ecosistemas.

\section{Composición y dominancia}

La composición de ecosistemas fue evaluada a partir de la clasificación de los usos de suelo para cada alternativa de localización. La dominancia fue estimada a través de la superficie total de paisaje ocupada. Los ecosistemas fueron caracterizados a través de su estructura vertical (tipo de estrato dominante: herbáceo, arbustivo y arbóreo) y horizontal (densidad del estrato dominante: denso, semi-denso, abierto). Las métricas de paisaje utilizadas fueron: (1) superficie ocupada por cada tipo de ecosistema (expresada en porcentaje del total de superficie terrestre evaluada) y, (2) número de parches de cada tipo (número de unidades de cada tipo de ecosistema).

\section{Conectividad estructural}

El análisis de la conectividad estructural se realizó a partir de los mosaicos del paisaje. Se definió como el grado en el cual el paisaje teóricamente permite o facilita flujos ecológicos, en especial de organismos de amplios rangos de hogar (e.g., carnívoros) que se mueven entre parches de hábitat. Para ello se utilizó el índice de cohesión (IC) que mide la conectividad física entre parches. El valor de IC se acerca a 0 cuando los parches son muy escasos en el paisaje total y, a la inversa, se incrementa cuando la proporción de parches aumenta en el paisaje total evaluado (Pauchard et al. 2006). Se calcula con Fragstats 3.3 sobre la imagen satelital clasificada. El IC representa un indicador de la conectividad del paisaje en términos físicos más que ecológicos. Ningún tipo de flujo ecológico fue medido.

Las métricas de paisaje fueron calculadas con ArcView ${ }^{\circledR} 3.2$ (ESRI) y Fragstats 3.3 , sobre la imagen clasificada. Cada una de las alternativas de relocalización fue evaluada a partir de una panorámica general de alrededor de 1500 ha y otra de 2600 ha, para evitar que los resultados fueran influidos por la superficie del mosaico, sirviendo como análisis de sensibilidad. En terreno se validó este análisis, las necesidades de ajustes y se verificó la inexistencia de cambios importantes entre la fecha de la imagen y la prospección en terreno (Enero 10-17, 2009).

\section{Valor ambiental}

En tanto, el valor ambiental de los ecosistemas se evaluó a partir de la clasificación de los ecosistemas y su estado sucesional, el cual fue calificado mediante dos indicadores: heterogeneidad y madurez del ecosistema. En este estudio, se usó la estructura vertical del bosque como medida de heterogeneidad, es decir, a la presencia de estratos herbáceos, arbustivos y arbóreos, y a la diversidad de especies. A mayor diversidad de especies y mayor diversidad de estratos el ecosistema es más heterogéneo. Para evaluar el estado de madurez, se utilizó tanto la forma de vida (hierbas, arbustos y árboles) de las especies presentes como las especies dominantes de la comunidad de plantas. Especialmente en el caso de los arbustos, el tipo de especies es un buen indicador, ya que algunas son típicamente colonizadoras y están en fases tempranas de desarrollo del matorral (postintervención), mientras que otras especies se asocian a fases tardías o más maduras (Aravena et al 2002). En bosques, un indicador del estado de madurez, es la altura de los árboles y el diámetro basal (Cruz et al. 2007). La evaluación de estos indicadores fue realizada a nivel descriptivo y cualitativo, y luego se procedió, en terreno, a realizar una verificación de la estructura vertical dominante, observándose la densidad de los estratos bajos del bosque y la diversidad de estratos y especies, como apoyo a los análisis realizados en gabinete.

\section{Integración en evaluación multicriterio}

Los resultados de la evaluación ecológica fueron integrados en una evaluación multicriterio, que incluyó criterios de riesgos, geomorfológicos, de potencial urbano, de requerimientos de infraestructura, de conectividad y ambientales. Para cada uno de los criterios se definió una escala de uno a cinco en la cual el valor uno corresponde a las condiciones óptimas para la urbanización y el valor cinco corresponde a las peores condiciones para la urbanización (Pontificia Universidad Católica de Chile 2009). En el caso de los criterios ecológicos, el valor uno corresponde a aquellas áreas en las cuales la urbanización generaría un bajo impacto sobre los ecosistemas silvestres presentes, áreas con alto grado de intervención o degradadas y/o áreas en las cuales es posible adaptar el trazado urbano para mantener la conectividad entre los ecosistemas silvestres existentes. En tanto, el valor cinco corresponde a áreas en las cuales la urbanización generaría altos impactos negativos sobre ecosistemas silvestres, áreas en donde predomina el bosque nativo y/o áreas en las cuales el trazado urbano disminuiría la conectividad entre ecosistemas silvestres presentes.

La metodología aplicada requiere la participación activa de los agentes sociales involucrados en la toma de decisiones, por ello se realizaron dos evaluaciones secuenciales: la primera al interior del equipo de trabajo y la segunda con los profesionales de los servicios públicos (Taller Contraparte Técnica; enero 16, 2009; Pontificia Universidad Católica de Chile 2009), siendo ambas integradas en una evaluación final. Para asignar los valores se utilizaron los resultados de los diagnósticos realizados por el equipo técnico en la primera fase del estudio. A partir de dichos resultados se generaron recomendaciones para la posible urbanización en cada uno de los sectores evaluados, las cuales fueron incorporadas como lineamientos de diseño en el Plan Maestro de la alternativa escogida tras la evaluación multicriterio. 


\section{RESULTADOS}

Los tipos de ecosistemas que constituyen el mosaico del paisaje, tras el análisis inicial de gabinete (Pontificia Universidad Católica de Chile 2009) y posterior validación en terreno, son cinco: pradera, matorral, bosque, terrenos sin vegetación (principalmente playas) y cuerpos de agua (Tabla 1). En los tres primeros se encuentran diferenciaciones internas dadas por las especies dominantes, el estado sucesional de los ecosistemas y la presencia de especies exóticas. La Tabla 2 muestra las especies más frecuentes registradas en terreno. Las áreas estudiadas no presentan cultivos agrícolas ni plantaciones forestales y la única área urbana es la ciudad de Chaitén, con su aeródromo y embarcadero.

La representación o dominancia de cada tipo de ecosistema sigue una misma tendencia en las tres localidades, dominando el bosque, seguido del matorral y finalmente la pradera con baja participación. No obstante, la relación entre estos tipos de ecosistemas muestra diferencias explicadas por una mayor proporción de bosques y una menor superficie relativa de matorrales, praderas y playas. Esto, dado que hacia el interior predominan las áreas boscosas muy poco intervenidas. E1 índice de cohesión presenta los valores más altos para el bosque en las tres localidades, mientras que el matorral muestra el valor más bajo en Bahía Pumalín y la pradera obtiene el valor más bajo en Fandango. En términos de la sensibilidad del análisis según la extensión del mosaico, se encontró que el patrón general del paisaje, para los tres aspectos evaluados, se mantiene en cada localidad. El detalle de ambos análisis es presentado en la Tabla 3, mientras que su distribución espacial se presenta en la Fig. 2. La evaluación no incluyó la extensión cubierta por el mar (cuerpos de agua), por no representar un ámbito de evaluación en el contexto de una urbanización, al ser objeto de un cuerpo normativo e institucional diferente.

TABLA 1

Tipos de ecosistemas que constituyen el mosaico del paisaje.

Ecosystem types that constitute the landscape mosaic.

\begin{tabular}{|c|c|}
\hline \multicolumn{2}{|c|}{ Tipos de ecosistemas } \\
\hline Pradera & Resulta, en la mayoría de los casos, del clareo del bosque para pastoreo. \\
\hline Matorral & $\begin{array}{l}\text { Predomina en sectores en que se ha removido el bosque (por tala o incendios principalmente) } \\
\text { y que están en fases tempranas de recuperación, en algunos casos conforman matorrales de ñadis } \\
\text { (suelos húmedos). }\end{array}$ \\
\hline Bosque & $\begin{array}{l}\text { Representa el estado más avanzado de la sucesión ecológica en el área de estudio, } \\
\text { estos pueden tener diferencias entre ellos, siendo bosques jóvenes o maduros. }\end{array}$ \\
\hline
\end{tabular}

TABLA 2

Especies más frecuentes en el área de estudio.

Most frequent species in the study area.

\begin{tabular}{lc}
\hline Estrato & Especies frecuentes \\
\hline Arbóreo & $\begin{array}{c}\text { Nothofagus nitida (Phil.) Krasser (coigüe de Chiloé), Drimys winterii J.R.Forst. \& G.Forst. (canelo), } \\
\text { Amomyrtus meli (Phil.) D.Legrand \& Kausel (meli), Luma apiculata (DC.) Burret (arrayán rojo). }\end{array}$ \\
Arbustivo & $\begin{array}{c}\text { Raukaua laetevirens (Gay) Frodin (traumén), Chusquea quila } \text { Kunth (quila), Fuchsia magellanica } \\
\text { Lam (chilca), Caldcluvia paniculata (Cav.) D.Don (tiaca), Sophora } \text { sp. (mayú), Berberis } \text { sp. (michay), } \\
\text { Lophosoria quadripinnata (J.F.Gmel) C.Chr. (ampe), Gunnera } \text { sp. (pangue), Juncus sp. (juncos), } \\
\text { entre otras. }\end{array}$
\end{tabular}




\section{Fandango}

El bosque es ampliamente dominante en la localidad (mayor a $80 \%$ ), seguido del matorral (aproximadamente $10 \%$ ), la pradera y la playa (ambos inferior al $10 \%$ ). Tanto el bosque como el matorral tienen un alto grado de conectividad (IC > 97), a diferencia de la pradera que está más fragmentada. Estos valores indican que los remanentes de bosque existentes están bien conectados entre sí, aunque los bosques costeros están aislados de aquellos situados hacia el oriente (Fig. 2). Estos últimos se ubican en las laderas de los cerros, son más antiguos, tienen un menor grado de intervención y, probablemente, soportan la mayor cantidad y diversidad de fauna de la zona. Los bosques costeros, están altamente alterados bajo su dosel, manifestándose en una menor diversidad, escasez de arbustos y un estrato herbáceo muy extenso. El paisaje muestra variados estados sucesionales secundarios, coexistiendo cercanamente especies colonizadoras con especies de estados más tardíos, no obstante, en el bosque se observan pocos árboles longevos. Las praderas son amplias debido a procesos de clareo del bosque para su ocupación con fines recreacionales (camping, uso de playa en el área costera; Fig. 2). Además, existe una práctica intensiva de extracción maderera selectiva.

Como resultado de la evaluación del paisaje, se recomienda que la urbanización en este sector priorice los sectores de pradera y matorral, ya que tienen una distribución y continuidad que facilita el trazado urbano. La intervención en el bosque debería mantener una franja en los bordes de esteros y quebradas por su mayor heterogeneidad a nivel arbustivo y debido a la presencia de ejemplares arbóreos de gran envergadura.

\section{TABLA 3}

Métrica del paisaje en cada una de las localidades evaluadas. n/e: no evaluado. Santa Bárbara (SB), Fandango (F) y Bahía Pumalín (BP). Entre paréntesis se presenta la relación porcentual entre los cuatro tipos de ecosistemas evaluados (bosque, matorral, pradera y playa), descontando la superficie de mar (cuerpos de agua).

Landscape metrics in locations evaluated. n/e: not rated. Santa Bárbara (SB), Fandango (F) and Bahía Pumalín (BP). Parentheses shows the percentage ratio between the ecosystem types evaluated (forest, scrub, grassland and beach), discounting the sea surface (water bodies).

\begin{tabular}{|c|c|c|c|c|c|c|c|}
\hline Métricas del paisaje & Tipo de ecosistemas & SB (2672 ha) & SB (1524 ha) & F (2649 ha) & $\mathrm{F}(1531$ ha) & BP (2651 ha) & BP (1517 ha) \\
\hline Participación porcentual & Bosque & $50.5 \%(77.0 \%)$ & $44.1 \%(71.7 \%)$ & $65.7 \%(85.8 \%)$ & $60.8 \%(81.3 \%)$ & $65.6 \%(87.8 \%)$ & $48.6 \%(81.0 \%)$ \\
\hline del tipo de ecosistema & Matorral & $11.1 \%(16.9 \%)$ & $11.9 \%(19.3 \%)$ & $6.1 \%(7.9 \%)$ & $8.1 \%(10.8 \%)$ & $3.9 \%(5.2 \%)$ & $3.3 \%(5.5 \%)$ \\
\hline \multirow[t]{3}{*}{ en el mosaico evaluado* } & Pradera & $1.9 \%(2.8 \%)$ & $2.0 \%(3.3 \%)$ & $3.1 \%(4.0 \%)$ & $3.9 \%(5.2 \%)$ & $1.1 \%(1.5 \%)$ & $1.6 \%(2.7 \%)$ \\
\hline & Sin vegetación (playas) & $2.1 \%(3.2 \%)$ & $3.5 \%(5.7 \%)$ & $1.7 \%(2.3 \%)$ & $2.1 \%(2.7 \%)$ & $4.1 \%(5.5 \%)$ & $6.4 \%(10.7 \%)$ \\
\hline & Cuerpos de agua (mar) & $34.4 \%$ & $38.50 \%$ & $23.40 \%$ & $25,20 \%$ & $25.30 \%$ & $40.00 \%$ \\
\hline \multirow[t]{5}{*}{ Número de parches } & Bosque & 46 & 33 & 43 & 35 & 16 & 14 \\
\hline & Matorral & 239 & 172 & 70 & 62 & 120 & 81 \\
\hline & Pradera & 48 & 24 & 60 & 46 & 21 & 17 \\
\hline & Sin vegetación (playas) & 8 & 8 & 15 & 7 & 38 & 35 \\
\hline & Cuerpos de agua (mar) & 1 & 1 & 1 & 1 & 1 & 1 \\
\hline \multirow[t]{5}{*}{ Índice de Cohesión } & Bosque & 99.8 & 99.6 & 99.9 & 99.8 & 99.9 & 99.8 \\
\hline & Matorral & 95.4 & 94.6 & 97.5 & 97.3 & 92.9 & 88.5 \\
\hline & Pradera & 93.6 & 95.4 & 94.7 & 94.4 & 93.6 & 93.8 \\
\hline & Sin vegetación (playas) & 96.9 & 96.8 & 96.2 & 96.5 & 96.7 & 96.2 \\
\hline & Cuerpos de agua (mar) & $\mathrm{n} / \mathrm{e}$ & $\mathrm{n} / \mathrm{e}$ & $\mathrm{n} / \mathrm{e}$ & $\mathrm{n} / \mathrm{e}$ & $\mathrm{n} / \mathrm{e}$ & $\mathrm{n} / \mathrm{e}$ \\
\hline
\end{tabular}



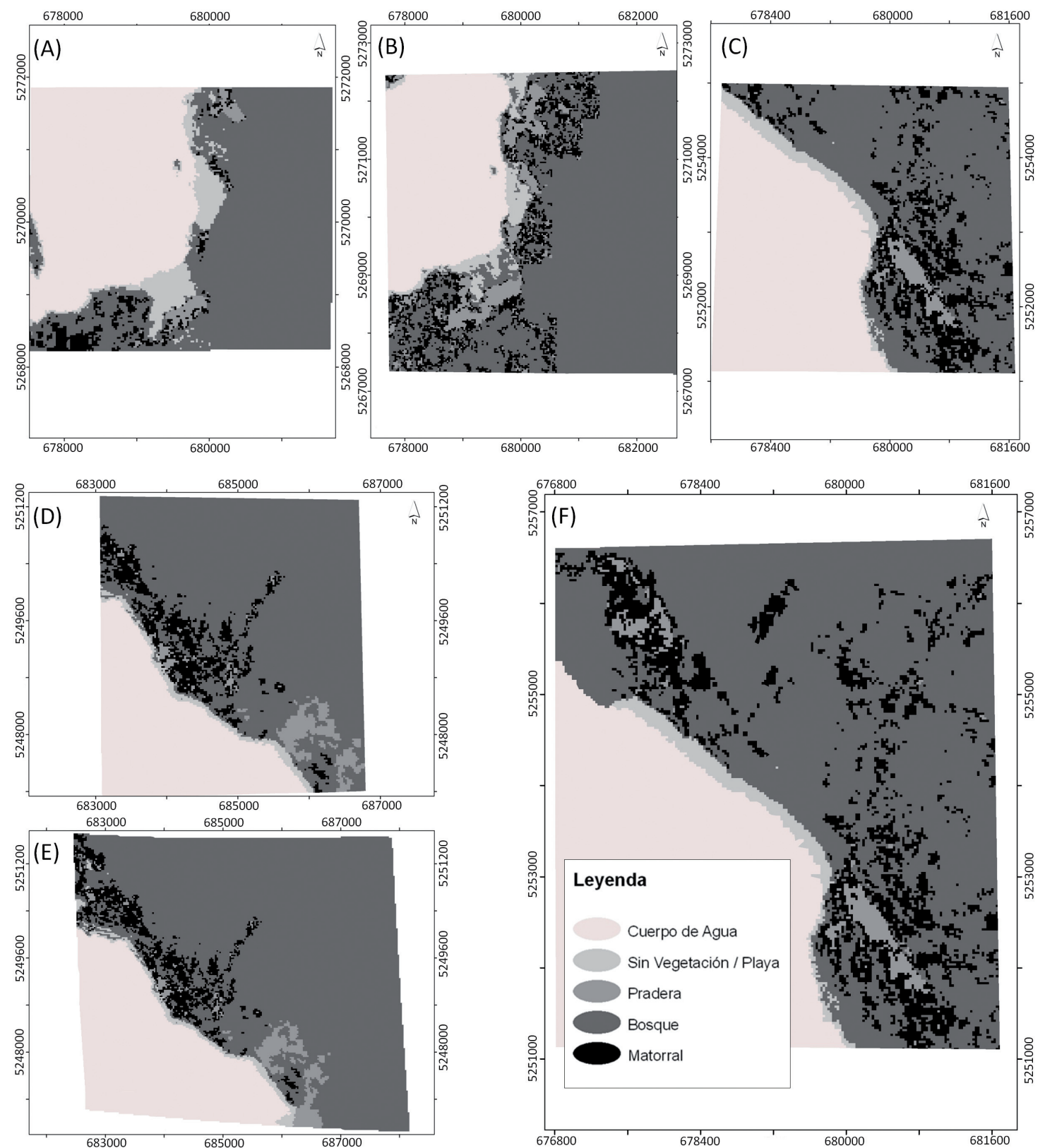

Fig. 2: Mosaicos de paisaje tras la clasificación de los tipos de ecosistemas dominantes en dos niveles de extensión. (A) Bahía Pumalín (mosaico de 2651 ha), (B) Bahía Pumalín (mosaico de 1517 ha), (C) Santa Bárbara (mosaico de 2672 ha), (D) Fandango (mosaico de 2649 ha), (E) Fandango (mosaico de 1531 ha), (F) Santa Bárbara (mosaico de 1524 ha).

Landscape mosaics after the classification of dominant ecosystem types for two extent levels. (A) Bahía Pumalín (2651 ha), (B) Bahía Pumalín (1517 ha), (C) Santa Bárbara (2672 ha), (D) Fandango (2649 ha), (E) Fandango (1531 ha), (F) Santa Bárbara (1524 ha). 


\section{Santa Bárbara}

Esta localidad presenta más del $70 \%$ de su superficie terrestre cubierta por bosque y, comparativamente, la mayor superficie cubierta por matorral (aproximadamente $18 \%$ ). A partir de los índices de cohesión, se observa que los bosques tienen un alto grado de conectividad en los dos mosaicos analizados (IC > 99), mientras que el matorral muestra un valor más alto que la pradera en el mosaico de mayor extensión, y más bajo en el mosaico de menor superficie (Tabla 3). Los bosques ubicados hacia el interior (Fig. 2) tienen un alto valor ambiental, por su estado maduro y alta heterogeneidad, formando bosque mixto de tres o cuatro especies que comparten la dominancia, presentan líquenes, abundantes plantas trepadoras y una mayor densidad en general. Este patrón se repite a nivel arbustivo con una baja alteración humana. En tanto, hacia el sector costero, el paisaje es dominado por praderas antropizadas (viviendas aisladas y uso ganadero hacia el sur) y matorrales (renovales), conservándose un bosque costero tardío de mediano a alto valor ecológico. Hacia el norte (ver Fig. 2), en el sector dominado por matorrales o bosques jóvenes, los suelos son propiamente de ñadis y la comunidad vegetal es bruscamente diferente, presentando algunas de las especies frecuentes en las otras unidades, pero agregando especies conspicuas como Podocarpus nubigenus Lindl. (mañío macho) -muy escasos-, Saxegothaea conspicua Lindl. (mañío) y Pilgerodendron uviferum (D.Don) Florin (ciprés de las guaitecas) en grupos bastante densos pero con baja presencia en el paisaje global, además de Weinmannia trichosperma Cav. (maden), Sophora sp. (mayú) y Embrothrium coccineum JR.Forst. \& G.Forst. (notro), entre otras, coincidiendo con la descripción de Lubert \& Pliscoff (2006).

La intervención del bosque es factible, si se evita la fragmentación del parche boscoso de mayor extensión ubicada en el oriente. En el sector costero, es importante mantener la continuidad de los fragmentos de bosque existentes (Fig. 2), dado que tiene una composición ligeramente diferente a los bosques situados hacia el interior (oriente). La urbanización en este sector debería priorizar los sectores de pradera y matorral, los cuales ya tienen un grado de ocupación humana. Para la urbanización en los sectores de cobertura boscosa se propone mantener la continuidad de los bosques, mediante la ocupación progresiva desde sectores aledaños a la Ruta 7 (que tienen mayor intervención; Fig. 1) hacia el oriente de dicho camino. Se recomienda mantener la integridad de las quebradas y sectores aledaños al estero Santa Bárbara, tanto por la presencia de especies vegetales características de las riberas y quebradas, como para el control natural de los incrementos de caudal.

\section{Bahía Pumalín}

En este sector predomina también el bosque (mayor a $80 \%$ ), mientras que el matorral y la pradera se concentran en la zona costera, asociados a una mayor intervención antrópica. La baja pendiente de la bahía determina una gran franja intermareal, de ecosistemas estuarinos y playas que ocupan una porción importante del paisaje (5.5 \% y $10.7 \%$ para mosaicos de 2651 y 1517 ha respectivamente). La continuidad del bosque es muy alta, siendo prácticamente una sola gran unidad (Tabla 3 ). La baja ocupación humana explica la presencia de ecosistemas de alto valor ambiental, maduros y heterogéneos.

Dada la integridad de los bosques y el bajo grado de intervención actual de los diversos ecosistemas presentes en Bahía Pumalín, una eventual urbanización debe localizarse en el margen del bosque ubicado al oriente (Fig. 2), sin afectar su integridad y manteniendo la continuidad a lo largo de quebradas y esteros. Asimismo, se debería mantener la continuidad del borde costero, especialmente de las extensas marismas, ya que constituyen una pieza importante de la dinámica de las especies marinas costeras, las cuales son especialmente vulnerables.

\section{Integración de los resultados con la evaluación multicriterio}

Los resultados del análisis ecológico fueron traspasados a la escala de valoración previamente definida para ser integrados en la evaluación multicriterio, junto con los resultados de las restantes áreas temáticas que abarcó el estudio de relocalización de 
Chaitén. Respecto del componente biodiversidad y ecosistemas, Fandango y Santa Bárbara obtuvieron un valor similar y cercano al óptimo para la urbanización (2), aunque con ciertas diferencias entre ellos (Pontificia Universidad Católica de Chile 2009). En Fandango, una eventual área urbana debería localizarse en el sector costero en donde se concentran las praderas y matorrales, para evitar el impacto negativo sobre los bosques maduros. La permanencia de algunos renovales de bosque nativo asegura una serie de servicios ecosistémicos, pero tiene una función ecológica reducida, ya que no afecta la continuidad de los bosques en la escala mayor y tiene una baja diversidad de especies, producto de la extracción selectiva y antropización del sector. En Santa Bárbara se registra una mayor diversidad del paisaje (playas, bosques, matorrales, praderas y estero) pero también un mayor grado de intervención antrópica. Presenta el mayor potencial de urbanización en los sectores ya cubiertos de praderas y matorrales, aunque se debe mantener la continuidad de los bosques maduros y de la vegetación aledaña al estero Santa Bárbara. En este caso, es posible adaptar el diseño de la urbanización y las redes de infraestructura para asegurar la continuidad de los bosques y ecosistemas ribereños.

Bahía Pumalín obtuvo el valor menos óptimo para la urbanización (5), porque presenta un bajo grado de intervención y una gran diversidad de ecosistemas, destacando la presencia de extensos sistemas estuarinos (Pontificia Universidad Católica de Chile 2009). Estos sistemas son muy sensibles a la intervención antrópica, lo cual explica la valoración asignada. Aunque sería posible emplazar la urbanización en las áreas cubiertas de praderas y matorrales, para disminuir su impacto negativo, las instalaciones de infraestructura (e.g., plantas de tratamiento de aguas, rellenos sanitarios, subestación eléctrica, caminos) afectarían a los sectores inundables y estuarinos, así como también a los bosques nativos que predominan en esta localidad.

La evaluación del equipo técnico y de los profesionales de servicios públicos coincidieron respecto del resultado final: Santa Bárbara representa el lugar óptimo para la relocalización de Chaitén, Fandango fue evaluado como un lugar medianamente adecuado y Bahía Pumalín tiene muy bajo potencial para la urbanización. Pero hubo algunas diferencias de valoración en criterios específicos. En el caso de los componentes ecológicos, los funcionarios públicos asignaron un mayor valor a los renovales de bosque nativo presentes en Fandango, sin diferenciarlos de los bosques maduros, y consecuentemente asignaron un valor menos óptimo para la urbanización (4) (Pontificia Universidad Católica de Chile 2009). E1 resultado final de esta evaluación multicriterio fue entregado a las autoridades políticas en el mes de enero de 2009.

\section{Incorporación de criterios ecológicos a la alter- nativa escogida}

El 25 de febrero de 2009 se anunció que Santa Bárbara es la localidad escogida para construir Nueva Chaitén. Los criterios de decisión destacados por la entonces Ministra de Vivienda y Urbanismo fueron aquellos relacionados con la conectividad vial, portuaria (nuevo puerto) y aérea (nuevo aeropuerto) (Ministerio de Vivienda y Urbanismo 2009). Respecto a las alternativas desestimadas, Fandango fue descartada por criterios relativos a la seguridad de las personas y de los asentamientos humanos, y Bahía Pumalín por su localización distante y desconectada de la red vial existente, situación que implica mayores costos y dificultades para una eventual urbanización (Pontificia Universidad Católica de Chile 2009).

En la decisión de localización de Nueva Chaitén, los aspectos ecológicos o ambientales estuvieron en segundo plano. No obstante, en el diseño del Plan Maestro de la Nueva Chaitén se incorporaron las recomendaciones ecológicas y ambientales correspondientes a la alternativa de Santa Bárbara, del modo que se indica en la Tabla 4. Ello se traduce en corredores verdes: áreas protegidas de bosque nativo integradas al área urbana que penetran en la ciudad, cuya estructura inicial consiste en cuatro sectores (centro cívico y tres barrios residenciales) separados por corredores naturales (quebradas y esteros) conectados a través de calles y parques (Pontificia Universidad Católica de Chile 2009). 


\section{DISCUSIÓN}

\section{De los patrones de intervención detectados}

Los resultados obtenidos indican que el patrón de fragmentación de los bosques en la Provincia de Chaitén es similar a lo descrito por Torres-Gómez et al. (2009) para la Provincia de Aysén, ya que los sectores más intervenidos son aquellos con menores pendientes, cercanos a la costa y adyacentes a los caminos existentes, lo cual es evidenciado por el reemplazo de la cobertura boscosa por praderas, renovales y agrupaciones de arbustos. Ello, porque las actividades agrícolas y ganaderas, predominantes en las zonas rurales de la región, utilizan terrenos más planos y accesibles, lo que determina que a menor pendiente se registre un mayor nivel de intervención (Torres-Gómez et al. 2009).

La distribución de los fragmentos de bosque nativo, sumado a las tendencias de uso del suelo en la región, permite esperar que los impactos potenciales sobre los bosques nativos sean ocasionados por las actividades económicas desarrolladas en zonas rurales planas o de baja pendiente. De esta manera, las medidas de mitigación deben enfocarse sobre

\section{TABLA 4}

Recomendaciones de la evaluación ecológica rápida y componentes del Plan Maestro de Nueva Chaitén. Fuente: Pontificia Universidad Católica de Chile (2009).

Santa Barbara's rapid ecological asssessment recommendations and Nueva Chaitén's Master Plan components. Source: Pontificia Universidad Católica de Chile (2009).

\section{Recomendación de la evaluación ecológica rápida Santa Componente del Plan Maestro}

Bárbara

Ajustar el diseño de la urbanización y las redes de infraestructura a las condiciones de conectividad ecológica locales.

Minimizar la fragmentación de las unidades de paisaje existente.

Priorizar la ocupación de los sectores más intervenidos, aledaños a la Ruta 7.

Mantener la vegetación de quebradas y riberas de los cursos de agua, por la presencia de especies sensibles y para el control natural de los incrementos de caudal.

Diseñar una trama urbana permeable a los diferentes componentes de la fauna y flora local que deben ser protegidos, conservados y conocidos por la comunidad local.

Mantener la integridad de los bosques situados al oriente de Santa Bárbara, por su madurez y alta heterogeneidad.

Conservar los fragmentos de bosque costero tardío (más maduro) situado en la ladera del cerro que marca el límite sur de Santa Bárbara.

Asegurar la permanencia de la comunidad vegetal presente en los suelos de ñadis, por su singularidad y diversidad.
El Plan Maestro mantiene la conectividad de los principales ecosistemas, y propone obras de infraestructura (aguas lluvia, calles, puentes) de bajo impacto.

La urbanización se concentra en sectores ya intervenidos (pozo de áridos, praderas) y se proyecta en etapas sucesivas.

La urbanización se inicia adyacente a la Ruta 7 con la instalación de servicios municipales y de Carabineros.

Se diseñaron áreas de preservación en las riberas de los cursos de agua, con ancho suficiente para mantener la diversidad vegetacional y favorecer la presencia de fauna.

Se proponen zonas de preservación en las riberas de los esteros, en el borde costero y en el límite oriente de la ciudad.

Se propone una Parque Natural en los cerros del oriente.

La urbanización se proyecta en el borde costero, con una segunda etapa hacia el interior, minimizando la intervención de estos bosques.

Este sector no se incluyó en el Plan Maestro como área urbanizable.

La urbanización proyectada ocupa una fracción de estos suelos e incluye en el sector norte el Parque del Ciprés de las Guaitecas. 
ellas. Ejemplo de esto será la infraestructura de transporte, que incluye la construcción de un nuevo puerto, un aeródromo, y el mejoramiento, ensanche y continuidad de la Ruta 7 con la carretera austral.

\section{De la evaluación ecológica y la eficacia de la estrategia metodológica}

La urbanización produce cambios radicales en la estructura de los hábitat y podría generar la extinción local de especies con problemas de conservación, dado que genera una transformación extrema de la estructura de la vegetación y de la composición de especies (Shochat et al. 2006). Por esta razón, para la evaluación de impactos ambientales en el marco del Sistema de Evaluación de Impacto Ambiental (SEIA), el énfasis en la evaluación de la biodiversidad se hace a nivel de especies y sus hábitats. Las especies tradicionalmente evaluadas y seleccionadas como objetos a conservar, son aquellas más conspicuas (flora vascular y fauna terrestre vertebrada) 0 aquellas con problemas de conservación, lo que podría tener consecuencias negativas para el resto de diversidad biológica, que no obstante, podría ser conservada si los ecosistemas (o paisajes) son conservados (Serey et al. 2007). Asimismo, una evaluación centrada en especies, requiere de instrumentos como líneas de base de flora y fauna, que describen principalmente la composición, escasamente la abundancia (ya que requiere mucho esfuerzo) y más exiguamente su distribución. Al requerir la incorporación de la variación estacional de las poblaciones, se alargan los tiempos de evaluación y con frecuencia se obtienen datos insuficientes, que exigen mucha interpretación. Por estas razones es que el análisis realizado sobre información de fácil acceso (estructura de la vegetación y estructura del paisaje) es pertinente para el caso. Otro factor a considerar es la escasez del conocimiento sobre cuáles son las especies presentes y cuáles tienen problemas de conservación a nivel regional y del territorio específico, lo que dificultaría un análisis de hábitat de especies objetivo. La metodología propuesta permite obtener una evaluación general, no obstante tiene limitaciones en caso de existir en el área especies de distribución restringida con bajos números poblacionales, situación en la cual se requieren estudios dirigidos. Así, en la EAE se diagnostica el estado del paisaje y se detectan especies especialmente sensibles de flora y fauna (e.g., anfibios o reptiles), para que después, cada intervención puntual en el territorio (e.g., construcción de infraestructura, urbanización) realice estudios detallados enfocados en especies, para la implementación de medidas específicas.

La estructura y edad del bosque son factores importantes para la diversidad de aves, invertebrados, anfibios y del estrato herbáceo; así como para presencia y actividad de murciélagos. Se correlacionan positivamente con la biodiversidad en ecosistemas de bosques y praderas por lo que representa un valioso indicador de la biodiversidad total de los ecosistemas (Ausden 2007, Adams et al. 2009). Así, los atributos escogidos en este estudio pueden ser rápidamente obtenidos representando buenos indicadores proxy de la heterogeneidad y diversidad del paisaje en contextos como en el que se realizó el estudio, caracterizado por escasa disponibilidad de información y urgencia en la obtención de resultados. La evaluación realizada fue dependiente de la percepción de los observadores, no obstante, tuvo un sentido comparativo entre categorías fácilmente diferenciables. Esto, dado que la metodología se restringe a ecosistemas terrestres donde el bosque representa el estado sucesional más avanzado y la heterogeneidad del paisaje es baja. Asimismo, si fuera posible contar con más tiempo y recursos, es recomendable usar otras métricas estructurales in situ.

Nueva Chaitén ocupará aproximadamente 20 ha en la primera etapa de construcción, de acuerdo con los planes elaborados por el Ministerio de Vivienda (Barrientos 2009). Una intervención de esta magnitud se expresa en la escala de paisaje causando la pérdida de hábitat, fragmentación y degradación de los ecosistemas existentes (Johnson \& Klemens 2005, Shochat et al. 2006). Por tanto, la evaluación de la composición y estructura del paisaje a escala 1:25000 es adecuada para el tipo de intervención analizado y, además, provee una línea de base para la evaluación posterior de los impactos generados por la nueva urbanización. Esto último, facilita también el diseño de acciones correctivas. No obstante, dependiendo del tipo de intervención 
específico a realizarse en áreas sensibles, puede ser necesaria y/o exigible por ley una evaluación más detallada, que permita la implementación de otras acciones preventivas, mitigadoras o correctivas específicas.

La función fue el único de los atributos del paisaje no evaluado. La función es descrita como las interacciones entre los elementos del paisaje, manifestándose como flujos de materia, energía, especies y/o información. Al respecto, el índice de cohesión fue usado como un indicador teórico de flujos ecológicos (e.g., organismos), ya que mide la conectividad física entre parches. Estos flujos pueden tener una expresión conceptual como servicios ecosistémicos, por cuanto aquello que fluye entre los ecosistemas puede generar condiciones y elementos de bienestar para la sociedad que habite en el paisaje. La razón de no evaluar este aspecto es que existe escaso conocimiento sobre la relación entre la heterogeneidad espacial y las consecuencias para los servicios ecosistémicos, representando una frontera de investigación, que puede ser de gran interés para el estudio de ciudades (Lovett et al. 2005).

\section{De la toma de decisiones y la normativa vigente}

El análisis de la composición y estructura del paisaje, y de las especies vegetales más conspicuas, presenta importantes ventajas como herramienta de evaluación rápida para la toma de decisiones: (1) Las unidades de paisaje (bosque, matorral, pradera, zona urbana), así como los usos de suelo (áreas agrícolas, urbanas e infraestructura), son atributos ampliamente conocidos y utilizados por profesionales de distintas disciplinas y por los habitantes de un territorio, de manera que es posible construir una base de información compartida y comprensible, condición indispensable para la toma de decisiones informada. (2) Las distintas unidades de paisaje pueden ser identificadas con relativa facilidad a partir de imágenes satelitales o fotografías aéreas, para luego ser analizadas mediante estudios de terreno. En Chile, los gobiernos regionales cuentan con imágenes satelitales, e incluso con restituciones aerofotogramétricas, lo cual constituye un valioso punto de partida para el análisis de paisaje. (3) Por último, las imágenes satelitales son cada vez más económicas, de mejor calidad y de fácil acceso. Todo ello es una condición importante para la toma de decisiones, que frecuentemente requiere análisis rápidos con insuficiente información disponible. Esta metodología, basada en imágenes LANDSAT, funciona adecuadamente a escala 1:25000. Para análisis de mayor resolución es necesario utilizar imágenes satelitales submétricas, o bien, fotografías aéreas, las que pueden ser más difíciles de conseguir.

Existe una tendencia a esperar que los resultados de los estudios ecológicos conduzcan a recomendaciones para la conservación de ciertos paisajes, mediante la delimitación de áreas silvestres protegidas (Miller \& Hobbs 2002). El enfoque adoptado en el presente estudio, al reconocer los distintos usos y coberturas del suelo como integrantes del paisaje, diferenciando zonas más intervenidas y zonas con valor de conservación, hace posible incluir criterios para la conservación fuera de las áreas silvestres protegidas existentes o que se puedan definir en el futuro. En este enfoque, la conservación de la biodiversidad nativa no se resuelve únicamente mediante áreas silvestres protegidas, sino que incorpora todas las unidades de paisaje mediante criterios generales que adoptan características particulares para cada uso de suelo (urbano, agrícola, forestal, infraestructura).

La normativa urbana vigente en Chile no favorece la incorporación de criterios ecológicos en los planes de urbanización, ya que no permite que los Planes Reguladores Comunales definan áreas destinadas a la conservación. Estas últimas deben ser definidas por las instituciones competentes: Ministerio de Medio Ambiente y Corporación Nacional Forestal. La evaluación ecológica rápida entrega elementos valiosos para que estas instituciones inicien estudios detallados para la delimitación de áreas que protejan los objetos de conservación elegidos, que pueden ser especies con problemas de protección o ecosistemas de interés (o alto valor ambiental).

Al momento de realizar este estudio, la Evaluación Ambiental Estratégica (EAE) no estaba reconocida en la normativa ambiental chilena, por lo que no era exigible, situación que ha cambiado tras la promulgación de la 
Ley 20417, que obliga a una EAE a todos los planes urbanos y de ordenamiento territorial. A través de la metodología aplicada por los autores en el caso de Chaitén, fue posible entregar criterios y lineamientos factibles de incorporar en la decisión de localización de una nueva ciudad y en su diseño urbano, contrastando los potenciales impactos sobre la biodiversidad de cada alternativa. De esta manera, constituye un aporte para futuras EAE's de instrumentos de planificación territorial, los cuales deben definir la distribución de las actividades humanas en un determinado territorio.

En cuanto a la localización, la metodología de evaluación utilizada priorizó la urbanización en áreas con mayor grado de intervención, las cuales en este caso, se asocian a las praderas y matorrales intervenidos. Respecto del diseño, los lineamientos definidos fueron: (1) conectividad de fragmentos de bosques, (2) permeabilidad del área urbana para las especies nativas, (3) mantención de la integridad de bosques más maduros, y (4) conservación de la vegetación de ñadis. Estos lineamientos de diseño pudieron ser incorporados en el Plan Maestro porque se traducen en atributos espaciales de la urbanización, por ejemplo, la continuidad de la vegetación de esteros y quebradas se traduce en parques a lo largo de los cursos de agua.

De especial interés es la situación que enfrentaron los tomadores de decisiones, la sociedad (directamente afectada y aquella menos afectada) y los científicos, caracterizada por una demanda social de conocimientos importante, la presión y urgencia de tomar decisiones con sustento científico por parte de las autoridades y la escasez de datos adecuados. Esta situación ha sido descrita para una gran diversidad de ámbitos, siendo denominada como ciencia postnormal, global, de modo II o de segundo orden (Quétier et al. 2008), que se caracteriza tanto por la elevada incertidumbre del sistema en evaluación (por deficiencia de datos, por ejemplo), como los riesgos derivados de las alternativas de decisión (consecuencias sobre la población, por ejemplo).

Los resultados de este trabajo permiten destacar las ventajas de la metodología propuesta, a saber: su aplicabilidad en diferentes tipos de paisajes, la accesibilidad de los recursos necesarios, incorporación de criterios ecológicos para la toma de decisiones en el ámbito del ordenamiento territorial y de la planificación urbana, la incorporación de recomendaciones para la protección de la escala de paisajes y ecosistemas, superando con ello la tendencia predominante (en las decisiones públicas) hacia la protección de especies singulares y, por último, la entrega de resultados rápidos y bien fundados.

AGRADECIMIENTOS: La presente publicación ha utilizado la información proveniente del estudio "Alternativas de relocalización y/o reconstrucción de la ciudad de Chaitén, Los Lagos, Chile”, realizado con financiamiento del Gobierno Regional de Los Lagos. Se agradece a la Comisión Nacional de Actividades Espaciales (CONAE), de la República Argentina, que proveyó gratuitamente la imagen satelital utilizada en el presente estudio. Los autores agradecen al Centro Nacional del Medio Ambiente CENMA, Chile, por financiar los costos de publicación de este artículo.

\section{LITERATURA CITADA}

ADAMS MD, BS LAW \& KO FRENCH (2009) Vegetation structure influences the vertical stratification of open and edge-space aerialforaging bats in harvested forests. Forest Ecology and Management 258: 2090-2100.

ARAVENA JC, MR CARMONA, CA PEREZ \& JJ ARMESTO (2002) Changes in tree species richness, stand structure and soil properties in a successional chronosequence in northern Chiloé Island, Chile. Revista Chilena de Historia Natural 75: 339-360.

AUSDEN M (2007) Habitat management for conservation: A handbook of techniques. Oxford University Press, New York.

BARRIENTOS N (2009) El futuro de la Nueva Chaitén. Plataforma Urbana. URL: http:// www.plataformaurbana.cl/archive/2009/11/28/ el-futuro-de-la-nueva-chaiten/ (accedido Enero 27, 2010).

BROWN K, WN ADGER, E TOMPKINS, P BACON, D SHIM \& K YOUNG (2001) Trade-off analysis for marine protected area management. Ecological Economics 37: 417-434

CARN S, J PALLISTER, L LARA, J EWERT, S WATT, A PRATA, R THOMAS \& G VILLAROSA (2009) The unexpected awakening of Chaitén volcano, Chile. Eos 90: 205-206.

CASTRO J \& D DINGWELL (2009) Rapid ascent of rhyolitic magma at Chaitén volcano, Chile. Nature 461: 780-783.

CORPORACIÓN NACIONAL FORESTAL \& COMISIÓN NACIONAL DE MEDIO AMBIENTE (1999) Catastro y evaluación de los recursos vegetacionales nativos de Chile. Santiago, Chile: CONAF-CONAMA.

CRUZ P, P HONEYMAN, A PEZO \& C SCHULZE (2010) Análisis de crecimiento de árboles maduros de lenga (Nothofagus pumilio) en bosques de la XII Región, Chile. Bosque 28: 18-24. 
DREVER M \& K MARTIN (2010) Response of woodpeckers to changes in forest health and harvest: Implications for conservation of avian diversity. Forest Ecology and Management 259: 958-966.

FOLCH A, O JORBA \& J VIRAMONTE (2008) Volcanic ash forecast-Application to the May 2008 Chaitén eruption. Natural Hazards Earth System Sciences 8: 927-940.

FORMAN R (1995) Land mosaics. The ecology of landscapes and regions. Cambridge University Press, Cambridge, UK.

GREZ A, J SIMONETTI \& R BUSTAMENTE (eds) (2006) Biodiversidad en ambientes fragmentados de Chile: Patrones y procesos a diferentes escalas. Editorial Universitaria, Santiago, Chile.

HAGAR J (2007) Wildlife species associated with nonconiferous vegetation in Pacific Northwest conifer forests: A review. Forest Ecology and Management 246: 108-122.

HENIG M, \& A WEINTRAUB (2006) A dynamic objective-subjective structure for forest management focusing environmental issues. Journal of Multi-Criteria Decision Analysis 14: $55-65$.

HERVÉ D (2010) Noción y elementos de la justicia ambiental: Directrices para su aplicación en la planificación territorial y en la evaluación ambiental estratégica. Revista de Derecho (Valdivia, Chile) 23: 9-36.

JOHNSON EA \& MW KLEMENS (2005) Impacts of sprawl on biodiversity. En: Johnson EA \& MW Klemens (eds) Nature in fragments: The legacy of sprawl: 18-53. Columbia University Press, New York

LAHDELMA R, P SALMINEN \& J HOKKANEN (2000) Using multicriteria analysis in environmental planning and management. Environmental Management 26: 595-605.

LARA L (2009) The 2008 eruption of the Chaitén volcano, Chile: A preliminary report. Andean Geology 36: 125-129.

LIU S, W PROCTOR \& D COOK (2010) Using an integrated fuzzy set and deliberative multicriteria evaluation approach to facilitate decision-making in invasive species management. Ecological Economics 69: 23742382.

LOVETT G, C JONES, M TURNER \& K WEATHERS (eds) (2005) Ecosystem function in heterogeneous landscapes. Springer-Verlag, NewYork.

LUEBERT F \& P PLISCOFF (2006) Sinopsis bioclimática y vegetacional de Chile. Editorial Universitaria, Santiago, Chile.

MALCZEWZKI J, R MORENO-SÁNCHEZ, L BOJORQUEZ \& E ONGAY-DELHUMEAU (1997) Multicriteria group decision-making model for environmental conflict analysis in the Cape Region, Mexico. Journal of Environmental Planning and Management 40: 349-374.

MILLER JR \& RJ HOBBS (2002) Conservation where people live and work. Conservation Biology 16 : 330-337.

MINVU (2009) Ministerio de Vivienda y Urbanismo. Ministra Poblete "Vamos a construir una mejor Chaitén”. Ministerio de Vivienda y Urbanismo. URL: http://www.minvu.cl/opensite_det 20090226170108.aspx (accedido Enero 27, 2010).

NARANJO J \& C STERN (2004) Holocene tephrochronology of the southernmost part $\left(42^{\circ} 30^{\prime}-45^{\circ} \mathrm{S}\right)$ of the Andean southern volcanic zone. Revista Geológica de Chile 31: 291-306.

NOSS R (1990) Indicators for monitoring biodiversity: A hierarchical approach. Conservation Biology 4 : 355-364.

OÑATE J, D PEREIRA, F SUÁREZ, J RODRÍGUEZ \& J CACHÓN (2002) Evaluación ambiental estratégica: La evaluación ambiental de políticas, planes y programas. Mundi-Prensa Libros, Madrid.

PAUCHARD A, M AGUAYO \& P ALABACK (2006) Cuantificando la fragmentación del paisaje: Las métricas y sus significados ecológicos. En: Grez A, J Simonetti \& R Bustamante (eds) Biodiversidad en ambientes fragmentados de Chile: Patrones y procesos a diferentes escalas: 17-40. Editorial Universitaria, Santiago, Chile.

PONTIFICIA UNIVERSIDAD CATÓLICA DE CHILE (2009) Consultoría para el desarrollo de lineamientos estratégicos de reconstrucción/ relocalización y plan maestro conceptual postdesastre Chaitén. Etapa 2: Análisis de alternativas. Ministerio de Vivienda y Urbanismo, Santiago, Chile.

QUÉTIER F, J STEWART, G CRUZ, C HAMEL, H MORALES-GROSSKOPF \& E TAPELLA (2008) Making ecological knowledge relevant for landuse decision makers. En: Tiessen H \& J Stewart (eds) Applying ecological knowledge to land use decisions. SCOPE Series. URL: http://www.icsus c o p e o r g/Lat e s t $\% 20 \mathrm{Ne} \mathrm{w} \mathrm{s/}$ LandUseDecisionsWEB.pdf (accedido Diciembre 28, 2010).

RAMÍREZ C, R MAC DONALD \& C SAN MARTÍN (1996) Uso forestal de los ecosistemas de "ñadi”: Riesgos ambientales de la transformación de suelos en la Región de Los Lagos. Ciencia y Ambiente (Chile) 12: 82-88.

SEREY I, F DE LA BARRERA \& D MOREIRA (2007) Biodiversidad en ecosistemas y paisajes a escala regional. En: Serey I, M Ricci \& C SmithRamírez (eds) Libro rojo de la Región de O’Higgins: 95-109. Corporación Nacional Forestal - Universidad de Chile, Rancagua, Chile.

SHOCHAT E, P WARREN, S FAETH, N MCINTYRE \& D HOPE (2006) From patterns to emerging processes in mechanistic urban ecology. Trends in Ecology and Evolution 21: 188-191

TORRES-GÓMEZ M, L DELGADO, VH MARÍN \& RO BUSTAMANTE (2009) Estructura del paisaje a lo largo de gradientes urbano-rurales en la Cuenca del Río Aysén (Región de Aysén, Chile). Revista Chilena de Historia Natural 82: 73-82. 